\title{
Erratum to: Salvage of failed hip osteosynthesis for fractures with modular hip prosthesis
}

\author{
Panagiotis Karampinas $^{1}$ - George Kollias ${ }^{1} \cdot$ John Vlamis $^{1} \cdot$ Eustratios A. Papadelis $^{1}$ • \\ Spiros G. Pneumaticos ${ }^{1}$
}

Published online: 16 May 2015

(C) Springer-Verlag France 2015

\section{Erratum to: Eur J Orthop Surg Traumatol \\ DOI 10.1007/s00590-015-1622-5}

In the original article, corresponding author's (Panagiotis Karampinas) given name has been published incorrectly as Panagiotis K. The correct given name should be Panagiotis.

The online version of the original article can be found under doi:10.1007/s00590-015-1622-5.

Panagiotis Karampinas

karapana@yahoo.com

George Kollias

georgekollias.mail@gmail.com

John Vlamis

jvlamis@email.com

Eustratios A. Papadelis

ppdelis@gamil.com

Spiros G. Pneumaticos

irosp@bcm.edu

1 III Orthopaedic Department, KAT Hospital, University of Athens Medical School, 2 Nikis Str., Kifissia, 14561 Athens, Greece 